\title{
Automated Post-fault Diagnosis of Power System Disturbances
}

\author{
Stephen D. J. McArthur, Member, IEEE, Euan. M. Davidson, Non-member
}

\begin{abstract}
In order to automate the analysis of SCADA and digital fault recorder (DFR) data for a transmission network operator in the UK, the authors have developed an industrial strength multi-agent system entitled Protection Engineering Diagnostic Agents (PEDA). The PEDA system integrates a number of legacy intelligent systems for analyzing power system data as autonomous intelligent agents. The integration achieved through multi-agent systems technology enhances the diagnostic support offered to engineers by focusing the analysis on the most pertinent DFR data based on the results of the analysis of SCADA.

Since November 2004 the PEDA system has been operating online at a UK utility. In this paper the authors focus on the underlying intelligent system techniques, i.e. rule-based expert systems, model-based reasoning and state-of-the-art multi-agent system technology, that PEDA employs and the lessons learnt through its deployment and online use.
\end{abstract}

Index Terms- Multi-agent systems.

\section{INTRODUCTION}

$\mathrm{T}$ he proliferation of monitoring equipment on transmission and distribution networks has resulted in an everincreasing amount of data being made available to utility engineers. Data provided by Supervisory, Control and Data Acquisition (SCADA) systems, Digital Fault Recorders (DFR), microprocessor-based protection relays with fault recording capabilities, travelling-wave fault locators, and circuit breaker condition monitoring systems, can aid engineers in making more informed, and potentially more profitable, power system operations and asset management decisions. However, there are a number of barriers to the effective use of this data:

- The raw data is often uninformative: information relating to plant health or the performance of the power system is implicit rather than explicit; and

- The volume of data, especially under storm conditions, renders manual analysis intractable.

The requirement for tools and techniques to automate the analysis of different types of power systems data has driven research into the use of artificial intelligence (AI) for the best part of two decades [1-12]. As important, although less fully explore in the literature, is the fact the all the data sets above are related; they all contain data relating to how the power system reacts to disturbances. When performing analysis manually, engineers tend to take a holistic approach by analysing all the available data. Consider the post-fault diagnosis process as carried out by engineers at SP PowerSystems, the transmission system operator in central and southern Scotland:

1. Engineers begin by identifying occurrence of disturbances or 'incidents' from SCADA data. Some aspects of protection operation can be validated from SCADA, e.g. missing protection alarms or inter-trip alarms may suggest that part of the scheme failed to operate.

2. Knowledge of the occurrence of an incident is used to focus the retrieval and analysis of fault recorder data. As recorders are triggered on a depression in voltage, a large number of recorders across the network may capture data. Engineers are primarily interested in the DFR data from the faulted circuit.

3. After all the fault records pertaining the incident have been retrieved the engineers analyse the data, classifying the type of disturbances, calculating the fault clearance time, determining an approximate fault location based on calculated impedance, and validating that protection operated as expected.

4. Travelling wave fault locator records are also retrieved for the faulted circuit and the distance to fault calculated.

5. Circuit breaker conditioning monitoring data may also be analysed to assess breaker operation.

The result of the process above is the comprehensive analysis of the reaction of the protection system to the disturbance or fault based on a range of data sources. However, data volumes make performing this process manually time-consuming and thus costly: automation is required.

Automating the analysis of a variety of data sets and then collating the results in a meaningful manner is complicated by a number of factors:

- Data are stored in a variety of proprietary formats in different systems in different physical locations;

- Different techniques are best suited to analysis of different data sets;

- The information resulting from one type of analysis can be used to focus subsequent analyses. For example, knowing when and on what circuit an incident has occurred, as derived from SCADA, allows DFR data to be partitioned and the most pertinent records identified based on time-of-capture and location. 
- Analysis tools and data sources are liable to change over time as new data sourced become available and analysis techniques ameliorated. In other words, there is a requirement for flexible and extensible software architectures.

When building a practical automated post-fault analysis system the authors' approach was to use multi-agent system (MAS) technology to integrate a set of industry-strength legacy intelligent systems. The choice of MAS technology was primarily motivated by the flexibility and extensibility it offers [10][11].

While multi-agent technology provides a framework for automating the entire post-fault analysis process, the quality of PEDA's analyses is predicated on the efficacy of the intelligent systems the agents encapsulate. This paper focuses on the underlying AI techniques that PEDA exploits, the stateof-the-art MAS technology employed, mechanisms for delivering information to engineers in an effective and timely manner, and the practical experience of deploying a system robust enough for use in the power industry.

The paper begins with a brief description of the PEDA system, which has been operating online at SP PowerSystems, since November 2004, before discussion of the individual analysis techniques.

\section{Protection Engineering Diagnostic Agents}

PEDA (fig. 1) was designed to automate steps 1-3 of the post-fault analysis process described in the introduction to this paper. To do so, PEDA employs the following agents:

- An Incident and Event Identification (IEI) Agent: this agent 'wraps' a rule-based expert system that identifies power system incidents, such as disturbances and switching operations, from 'live' SCADA data. When an incident occurs the IEI agent uses the expert system to identify the relevant alarms, classify the type of incident and assess some aspects of protection operation.

- Fault Record Retrieval (FRR) Agents: the FRR agent connects to the utility's fault record database and makes new records available to other agents.

- A Fault Record Interpretation (FRI) Agent: the FRI agent 'wraps' a rule-based expert system for classifying and interpreting DFR data.

- A Protection Validation and Diagnosis (PVD) Agent: the PVD agent 'wraps' a model-based reasoning engine that employs models of the protection system to validation protection performance.

- A Collation Agent (CA): The collation agent (CA) gathers information from the agents above and archives it in a database accessible via an intranet site. This intranet site gives engineers access to the latest information provided by the PEDA agents and also allows them to explore historical data for particular circuits as well as download and view fault records on their local desktop PC.

- Engineering Assistant Agents (EAA): PEDA uses engineering assistant agents to provide tailored post-fault analysis information to engineers.

The agents automate post-fault diagnosis through social interactions. When a fault record is retrieved via auto polling, the FRR agent sends a message asking the IEI agent if any incidents occurred at the time the record was captured. The IEI agent then sends the details of any incidents that occurred at that time to the FRR agent. The FRR agent can then classify how the record is related to the incident. Records can be classified as:

- Directly related to an incident: a fault record captured during an incident from the end of the circuit involved in that incident.

- Related to an incident: a fault record captured during an incident from a substation that contains one of the ends of the circuit involved in the incident, however the record does not contain data from that particular circuit.

- Indirectly related to an incident: a fault record captured during an incident by a fault recorder in substation that was not directly involved in the incident. The fault recorder has triggered or been cross-triggered because of the voltage depression created by the disturbance.

- Miscellaneous fault record: a fault record that cannot be associated with an incident, i.e. no incidents occurred at the time the record was captured.

By performing the first-cut classification above, it is possible to quickly identify and highlight the fault records that are of immediate interest to protection engineers, i.e. records that are directly related to incidents, without having to analyze the content of the record.

The FRR agent then informs the FRI agent of the existence new record. The FRI and PVD agents can then perform their analysis and archive their results with the Collation Agent. Full details of these agent interactions can be found in [13] [14].

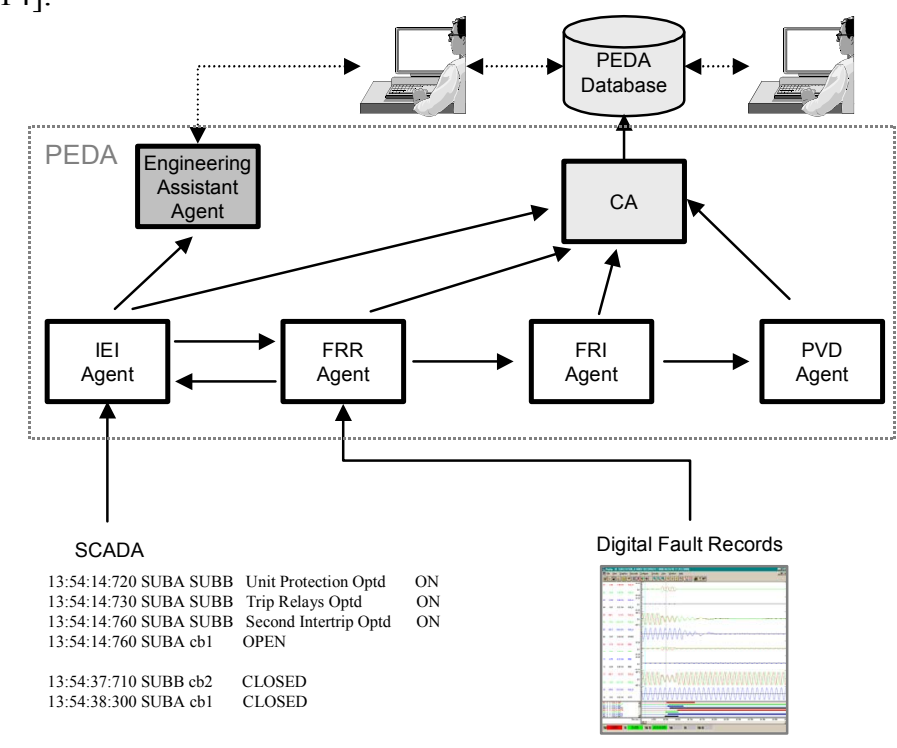

Figure 1: The PEDA multi-agent system

The agents were implemented using the Java Agent 
Development Environment [15] (JADE) and adhere to Foundation for Intelligent Physical Agents (FIPA) standards [16]. These standards provided a common agent communication language (FIPA-ACL) and an 'open' system architecture, allowing agents to be added and removed as and when required. They also promote interoperability with other multi-agent systems.

Ultimately, PEDA offers the protection engineer a list of power systems incidents derived from SCADA data, along with the interpreted DFR data and model-based analysis of any protection operation for each of the incidents. Through the PEDA intranet site, engineers can browse through analyses, make amendments and corrections, and get access to the raw data should it be required.

Historical data illustrates the effectiveness of the PEDA approach. In 2003 the fault recorder network captured 20,000 fault records yet only 66 disturbances occurred over the course of the year. Through the automated analysis of over 3 million SCADA alarms, PEDA was able identify the 66 incidents and partition the DFR data to identify the 1800 records of most importance before carrying out the in depth analysis using the FRI and PVD agents. Tests with historical data have also illustrated PEDA's ability to analyse data during storm conditions. For example, a storm in early 2002 caused a major disruption of supply in central Scotland. The SCADA system generated 15,000 alarms and the DFR network captured 1695 fault records in a 24-hour period. PEDA correctly identified 166 incidents from the SCADA, the majority occurring in a 4-hour period, and partitioned the DFR data accordingly [13].

Since November 2004 PEDA has been operating online at the utility. The results of the online trial of PEDA have already been reported in the literature [14]. These initial results illustrate the effectiveness of the approach and the robustness of the agents developed using agent development toolkits [17], a robustness that, as described in [13], was previously lacking.

While MAS technology provides a framework for building complex hybrid intelligent systems, PEDA's success is predicated on the underlying intelligent systems the agents encapsulate.

\section{AlARM PROCESSING FOR INCIDENT AND EVENT IDENTIFICATION}

PEDA's IEI agent uses a legacy alarm processor, entitled the Telemetry Processor, to automate the analysis of SCADA data. The Telemetry Processor was developed using a classic knowledge engineering approach. Knowledge was elicited from the utility's engineers of how they analysed SCADA data after a major disturbance. Fig. 2 illustrates the multi-stage process they employed:

Stage 1 - Identify the incident circuit and inception time by scanning the alarms for a pattern indicating incident inception, i.e. protection relay operation followed by a circuit breaker opening.
Stage 2 - Group the incident alarms by selecting the next alarm after incident inception which has not already been checked. Perform a topology check of the alarm against the incident circuit recording a matching alarm as part of the incident.

Stage 3 - Scan the already grouped alarms for patterns indicating incident conclusion, e.g. the end of a DAR sequence. If no conclusion is identified move back to stage 2 . This iterative process continues until the incident has been concluded.

Stage 4 - Scan the grouped incident alarms to identify which protection scheme components operated and when. Each operation is recorded as a low-level event and an event summary generated.

Stage 5 - The grouped alarms and low-level events are then analysed to group related events and identify whether all expected low-level events occurred. The results are recorded as high-level event summaries.

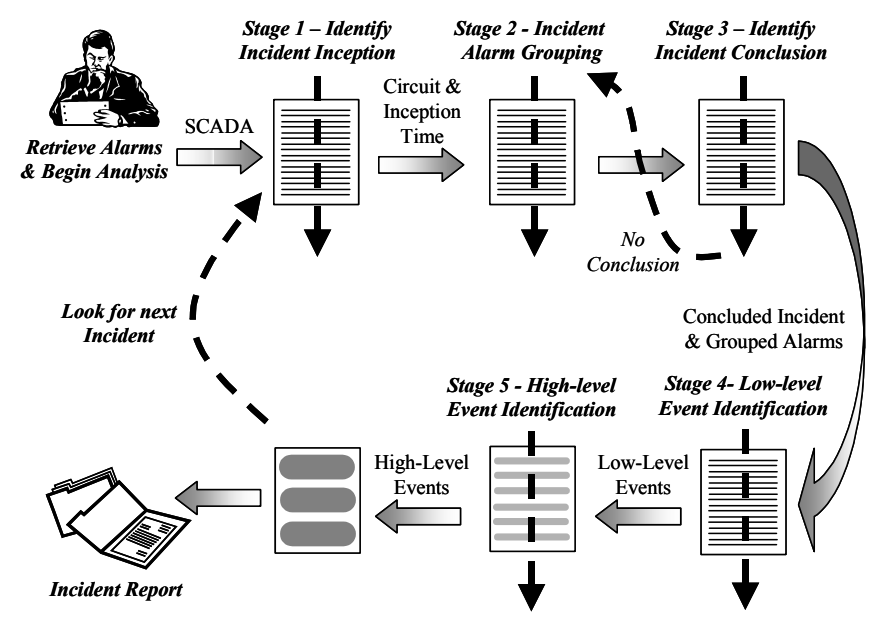

Fig. 2. Manual analysis of SCADA data.

The Telemetry Processor automates the analysis process above using the knowledge elicited from engineers. It adopts a reasoning technique employing multiple reasoning streams (Fig. 3). Each reasoning stream is implemented as an inference engine using knowledge appropriate to each alarm analysis stage. The knowledge is stored as production rules in separate knowledge bases to allow easy maintenance.

A single inference engine is permanently devoted to stage 1 with additional inference engines being created for each identified incident inception to manage stages 3 to 5 . These incident inference engines are transient and only exist until alarm interpretation has concluded for the identified incident. To manage the multiple inference engines and perform stage 2 the overall reasoning technique is separated into a control and inference layer as illustrated in Fig.3. The control layer controls each inference engine independently, supplying data to and retrieving data from each inference engine.

The Telemetry Processor was implemented using Java programming language and the JESS expert system shell [18]. The output, an incident report (Fig. 4) containing details of the incident, i.e. all the events which occurred during the incident 
and the high level summary generated in stage 5 , is stored in a database which engineers can access using a web browser via the corporate intranet.

Importantly, the Telemetry Processor operates online. Engineers, on the other hand, analysed the data after all the alarms had been received and placed in the correct order. As the Telemetry Processor has to deal with a 'live' SCADA feed, there was a knowledge gap that needed to be bridged in order to deal with data skew and the ordering of alarms.

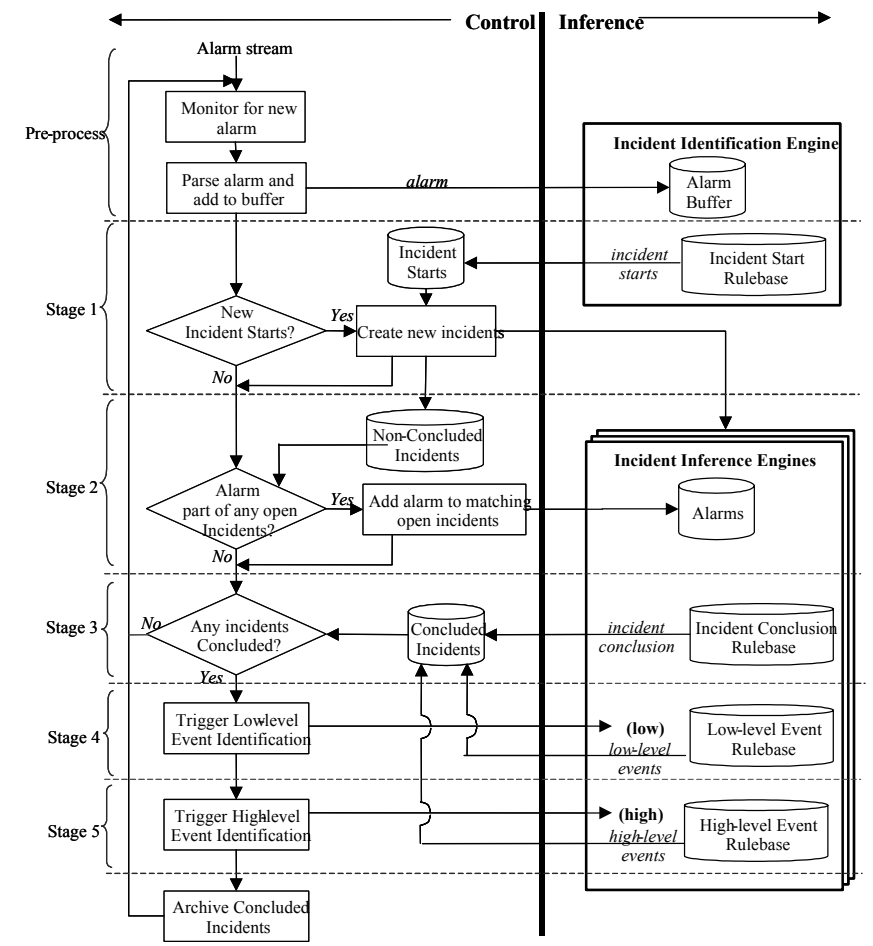

Fig. 3. Telemetry Processor Reasoning Engines

\begin{tabular}{|c|c|}
\hline Incident: & $\begin{array}{l}\text { "DISTANCE PROT OPTD - SUBB } / \text { SUBA: Autoreclose Complete" } \\
\text { START TIME = 14:20:39.07 } \\
\text { FINISH TIME }=14: 20: 39.28\end{array}$ \\
\hline Low-Level & $\begin{array}{l}\text { 14:20:39.07 "Distance protection operated ON at SUBB -> SUBA" } \\
\text { 14:20:39.10 "Autoreclose in progress at SUBB CB2" } \\
\text { 14:20:39.13 "SUBB CB2 OPEN" } \\
\text { 14:20:39.16 "Distance protection operated OFF at SUBB -> SUBA" } \\
\text { 14:20:39.21 "1st Intertrip received ON at SUBA from SUBB" } \\
\text { 14:20:39.21 "2nd Intertrip received ON at SUBA from SUBB" } \\
\text { 14:20:39.22 "SUBA circuit breaker CB3 OPEN" } \\
\text { 14:20:39.23 "Autoreclose in progress at SUBA CB1" } \\
\text { 14:20:39.24 "SUBA circuit breaker CB1 OPEN" } \\
\text { 14:20:39.26 "Autoreclose in progress at SUBA CB3" } \\
\text { 14:20:39.28 "Autoreclose sequence at SUBB CB2 took } 180 \mathrm{~ms} \\
\text { 14:20:39.28 "Autoreclose sequence complete at SUBB CB2" }\end{array}$ \\
\hline High-Level & $\begin{array}{l}\text { 14:20:39.07 "Distance protection operated successfully at SUBB -> SUBA } \\
\text { 14:20:39.21 "1st and } 2^{\text {nd }} \text { Intertrips received at SUBA from SUBB" } \\
\text { 14:20:39.21 "No protection operation at SUBA on circuit SUBB" } \\
\text { 14:20:39.28 "Unit protection at SUBB -> SUBA failed to operate" }\end{array}$ \\
\hline
\end{tabular}

Fig. 4. Telemetry Protection output

The Telemetry Processor was installed at SP PowerSystems as a standalone system in July 2003. It processes the SCADA data as soon as it arrives from the Energy Management System (EMS). In a typical day, it deals with between 10,000 and 20,000 alarms. In terms of speed, incident information was made available to engineers over the corporate intranet within one or two minutes of the conclusion of an incident.

By the time the Telemetry Processor was incorporated into the PEDA architecture as the IEI agent, there was already in excess of a year's operational experience with the system.

\section{EXPERT SYSTEMS FOR FAULT RECORD INTERPRETATION}

Over the last 15 years a number of different approaches to automating the analysis of DFR data have appear in the literature [6][7]. Most common is the rule-based expert system approach as typified by the work of Kezunovic et al [7]. Many manufacturers already offer products for the automatic classification of fault records.

The FRI agent encapsulates an expert system for classifying fault records that the authors developed as part of a previous project. The expert system reads in COMTRADE data and extracts the following information from the record:

- The fault classification, e.g. red-blue fault;

- The fault inception time; and

- The fault clearance time.

This expert system also transforms COMTRADE data into the format required by the model-based reasoning engine described in the next section.

\section{VAlidating Protection Operation USING MOdel- BASED REASONING}

In recent years engineers and researchers have developed a substantial number of protection relay models and tools for modelling [19][20][21]. A review of the application of relay models by the IEEE Systems Protection Sub-committee of the PSRC [19] suggested that these models could be used for troubleshooting. By propagating DFR data through an appropriate model of the relay in question and then comparing the results with the actual behaviour of the relay, engineers can confirm whether or not protection operated correctly.

Earlier research by McArthur et al [8][9] demonstrated how this model-based process of evaluating protection behaviour could be automated using model-based reasoning (MBR) techniques. The General Diagnostic Engine (GDE) [22] was extended to validate protection operation [12].

PEDA's PVD agent encapsulates a model-based reasoning engine [12] that supports the use of existing protection models, created and running on different modelling and simulation packages, for diagnosis (Fig. 5). At the time of writing the tool-set allows the integration of SIMULINK and MATLAB models, Dynamic Protection Models [20] and models written in $\mathrm{C}++$ and Java.

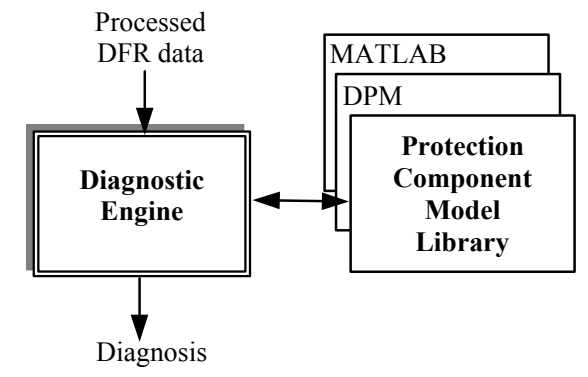

Fig. 5. The diagnostic engine draws on a library of protection component models in order to validate protection performance 


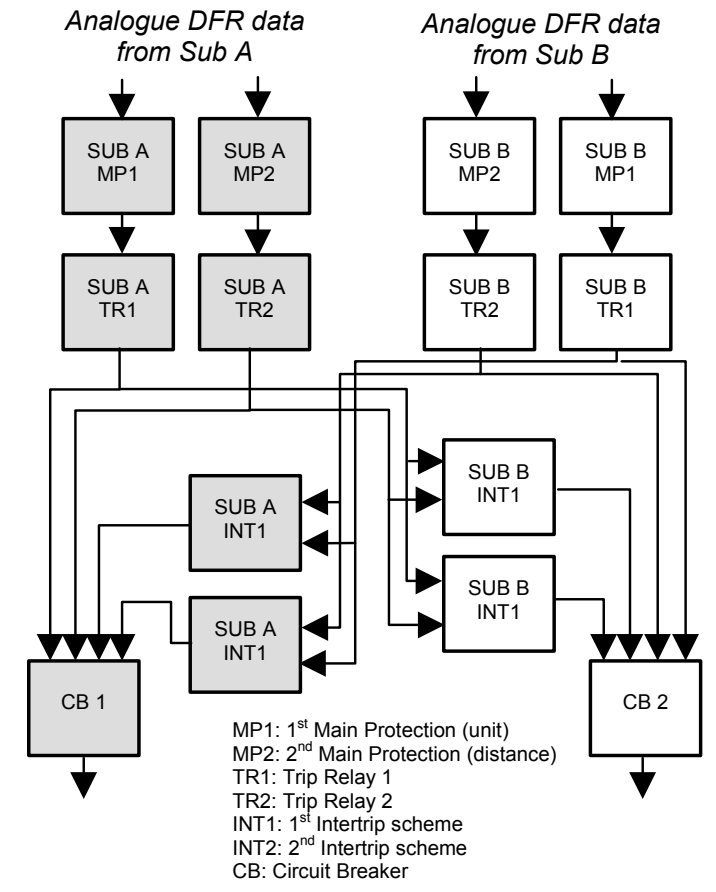

Fig. 6. Example protection scheme model

The diagnostic function is achieved by detecting deviation from nominal behaviour and identifying components of the system whose failure could be logically responsible for the deviation. To do this the MBR engine uses a model of structure (Fig. 6) and library of models which simulate the behaviour of different protection components. Full details of the algorithms the diagnostic engine employs can be found in [8][9][12].

The MBR approach has a number of advantages. As diagnosis is based on knowledge of function rather than malfunction, novel faults, i.e. faults never experienced before, can be diagnosed. The failure of more than one component of the protection scheme is also considered.

As described above, it allows the reuse of existing protection models. The technique is also model agnostic. It can employ a range of models from simple models capturing static relay characteristics to complex dynamic models with model the relay at the circuit board level [20].

Ultimately the MBR engine, and thus the PVD agent which encapsulates it, produces a protection validation report. The protection validation report gives details of the records analysed at the final diagnosis. An example diagnosis is, "Trip Relay 2 in Substation A failed to operate".

\section{CONCLUSIONS}

Multi-agent system technology provides a framework for creating open, extensible, distributed hybrid intelligent systems. Recent results have demonstrated that MAS technology has matured to the point where meaningful applications can be developed and installed at utilities. Nearly 2 years of online experience with the PEDA system is a case in point. MAS technology, however, is not a panacea for dealing with monitoring data. Classic intelligent systems tools and techniques, which had to be tailored for use in the power industry, underpin the data analysis activities agents can perform.

The benefit of the use of agents is that utilities can use this technology to build complex data analysis system which give a more holistic view of power system operation and performance based on all the available data.

\section{REFERENCES}

[1] D.B. Tesch, D.C. Yu, L-M. Fu, K. Vairavan, "A Knowledge-Based Alarm Processor for and Energy Management System", IEEE Transactions on Power Systems, v5, n1, February 1990, pp 268-275.

[2] Z.A. Vale, A.Machado e Moura, M. Fernando Fernandes, A. Marques, "SPARSE - An Expert System for Alarm Processing and Operator Assistance in Substations Control Centers", Applied Computing Review, v2, n2, 1994, pp 18-26.

[3] D.S. Kirshen, B.F. Wollenberg, "Intelligent Alarm Processing in Power Systems", Proceedings of the IEEE, v80, n5, May 1992, pp 663-672.

[4] J.R. McDonald, G.M. Burt, D.J. Young, "Alarm Processing and fault diagnosis using knowledge based systems for transmission and distribution network control", IEEE Transactions on Power Systems, v7, n3, pp 1292-1298, August 1992.

[5] J. Jung, C-C. Liu, M. Hong, M. Gallanti, G. Tornielli, "Multiple Hypotheses and Their Credibility in On-Line Fault Diagnosis", IEEE Transactions on Power Delivery, v16, n2, April 2001.

[6] L. Cederblad, P.O. Gjerde "A Knowledge-based System for the Automatic Evaluation of Disturbance Recordings" CIGRE, Paris, Paper $34-204$, August 1992

[7] M. Kezunovic, C.W. Fromen, D.R. Sevcik "An Expert System for Transmission Substation Event Analysis" IEEE Trans. on Power Delivery, Vol. 8, No. 4, pp1942-1949, October 1993

[8] S.D.J. McArthur, A. Dysko, J.R. McDonald, S.C. Bell, R. Mather, and S.M. Burt. "The Application of Model-based Reasoning within a Decision Support System for Protection Engineers" IEEE Transactions on Power Delivery, Vol. 11, No.4, pp. 1748 - 1755, October 1996

[9] S.C. Bell, S. D. J. McArthur, J. R. McDonald, G. M Burt, R. Mather, T. Cumming. "Model-based analysis of protection system performance" IEE Proceedings: Generation, Transmission and Distribution, v 145, n 5, pp. 547-552, September 1998

[10] J. A. Hossack, J. Menal, S. D. J. McArthur, J. R. McDonald, "A multiagent architecture for protection engineering diagnostic assistance", IEEE Transactions on Power Systems, Volume 18, Issue 2, May 2003, pp. $639-647$.

[11] S.D.J McArthur, J. R. McDonald, J. A. Hossack, "A Multi-Agent Approach to Power System Disturbance Diagnosis" in Autonomous Systems and Intelligent Agents in Power System Control and Operation (Power Systems), Ch Rehtanz (ed.), Springer-Verlag, July 2003, pp 7599.

[12] E. M. Davidson, S. D. J. McArthur, J. R. McDonald, "A toolset for applying model-based reasoning techniques to diagnostics for power systems protection". IEEE Transactions on Power Systems, Volume 18, Issue 2, May 2003, pp. $680-687$

[13] E. M. Davidson, S. D. J. McArthur, J. R. McDonald, , T. Cumming, I. Watt, "Automating the analysis and management of Power System Data using Multi-agent Systems Technology" Application and Innovations in Intelligent Systems XII (AI2004), Springer 2004, pp. 151-164

[14] E. M. Davidson, S. D. J. McArthur, J.R. McDonald, T. Cumming, I Watt. "Applying Multi-agent System Technology in Practice: Automated Management and Analysis of SCADA and Digital Fault Recorder Data" IEEE Trans. on Power Systems (in press)

[15] Java Agent Development Framework (JADE): Available: http://jade.cselt.it/

[16] Foundation for Physical Intelligent Agents (FIPA): http://fipa.org.

[17] E. E. Mangina, "Review of software products for multi-agent systems" Report: available from www.agentlink.org

[18] E.J. Friedman-Hill, "Jess, The Java Expert System Shell", http://herzberg.ca.sandia.gov/jess/, version 6.1a5, $15^{\text {th }}$ January 2003.

[19] P.G. McLaren, C. Henville, V. Skendzic, A. Girgis, M. Sachdev, G. Benmouyal, K. Mustaphi, M. Kezunovic, Lj. Kojovic, M. Meisinger, C. Simon, T. Sidhu, R. Marttila, D. Tziouvaras, "Software Models for 
Relays" IEEE Transactions on Power Delivery Vol. 16, No. 2, pp. 238246, April 2001

[20] A. Dysko, J.R. McDonald, G.M. Burt, J. Goody, B. Gwyn, "Integrated Modeling Environment A Platform For Dynamic Protection Modelling And Advanced Functionality", IEEE Power Engineering Society Transmission and Distribution Conference, April 1999

[21] M. Kezunovic, S. Vasilic, "Advanced Software Environment for Evaluating the Protection Performance Using Modeling and Simulation," CIGRE SC 34 Colloquium Sibiu, Romania, September 2001.

[22] J. de Kleer, B.C. Williams, "Diagnosing Multiple Faults", Artificial Intelligence, Vol. 32, No. 1, pp. 97-130, April 1987

\section{BIOGRAPHIES}

Dr Stephen McArthur is a Lecturer within the Institute for Energy and Environment. He received his B.Eng. (Hons) and $\mathrm{PhD}$ degrees from the University of Strathclyde in 1992 and 1996 respectively. His research interests include Intelligent System Applications in Power Engineering, Hybrid Intelligent Systems and Intelligent Agent Technology. Dr McArthur has 30 technical publications and is the co-editor of a book published by Chapman and Hall.

Euan Davidson holds a post as a Research Fellow at the Institute for Energy and Environment. He received his MEng. degree from the University of Strathclyde in 2000. His research interests include application of Model-based Reasoning and other artificial intelligence techniques in Power Engineering. 